\title{
Center of Gravity Estimation Using a Reaction Board Instrumented With Fiber Bragg Gratings
}

\author{
Rui OLIVEIRA ${ }^{1}$, Paulo RORIZ ${ }^{1,2}$, Manuel B. MARQUES ${ }^{1,3}$, and Orlando FRAZÃO ${ }^{1 *}$ \\ ${ }^{1}$ INESC TEC, Rua do Campo Alegre 687, 4150-179 Porto, Portugal \\ ${ }^{2}$ CIDESD-ISMAI -Institute University of Maia (ISMAI), Av. Carlos Oliveira Campos, Castelo da Maia, 4475-690 Maia, \\ Portugal \\ ${ }^{3}$ Department of Physics and Astronomy, Faculty of Sciences, University of Porto, Rua do Campo Alegre 687, 4169-007, \\ Porto, Portugal \\ ${ }^{*}$ Corresponding author: Orlando FRAZÃO \\ E-mail: ofrazao@fc.up.pt
}

\begin{abstract}
The purpose of the present work is to construct a reaction board based on fiber Bragg gratings (FBGs) that could be used for estimation of the 2D coordinates of the projection of center of gravity (CG) of an object. The apparatus is consisted of a rigid equilateral triangular board mounted on three supports at the vertices, two of which have cantilevers instrumented with FBGs. When an object of known weight is placed on the board, the bending strain of the cantilevers is measured by a proportional wavelength shift of the FBGs. Applying the equilibrium conditions of a rigid body and proper calibration procedures, the wavelength shift is used to estimate the vertical reaction forces and moments of force at the supports and the coordinates of the object's CG projection on the board. This method can be used on a regular basis to estimate the CG of the human body or objects with complex geometry and density distribution. An example is provided for the estimation of the CG projection coordinates of two orthopaedic femur bone models, one intact, and the other with a hip stem implant encased. The clinical implications of changing the normal CG location by means of a prosthesis have been discussed.
\end{abstract}

Keywords: Optical fiber sensors; fiber Bragg gratings; cantilever; center of gravity; hip prosthesis

Citation: Rui OLIVEIRA, Paulo RORIZ, Manuel B. MARQUES, and Orlando FRAZÃO, "Center of Gravity Estimation Using a Reaction Board Instrumented With Fiber Bragg Gratings," Photonic Sensors, 2018, 8(1): 1-6.

\section{Introduction}

The center of gravity (CG) is a point in space where the weight of a body is concentrated. Finding the coordinates of the CG has many applications, namely in biomechanics. However, the location of the CG of the whole body, a body segment (e.g., arm, forearm, and others), a prosthesis or other objects that interact with the human body could be a demanding task due to their complex geometry and the heterogeneity of the materials that compose them. Moreover, in the case of the body segments, which are connected through the joints in a continuous fashion, accurate measurements such as of the mass or weight of a single segment could be a cumbersome task because the segment ends or boundaries are not clearly distinguishable. In fact, depending on the assumptions used to define the segment landmarks the CG location could change [1, 2]. Several methods have been proposed in

Received: 13 September 2016 / Revised: 20 December 2017

(C) The Author(s) 2017. This article is published with open access at Springerlink.com

DOI: $10.1007 / \mathrm{s} 13320-017-0381-9$

Article type: Regular 
biomechanics to estimate the inertial properties of heterogeneous bodies and with complex geometry [3, 4]. The reaction-board method, also known as the "moment table", is one of them. It was firstly proposed by Reynolds e Lovett in 1909 with the purpose of estimating the location of the CG of the human body or the body parts [5] and immediately used in biomechanical and clinical applications [6] up to the present days [7]. The previous method consisted of a rectangular reaction-board supported at the ends by two knife edges horizontally leveled, one of them mounted on a scale, and the other a pivot point. Knowing the board's weight and length, along with the weight of the subject and the scale reading, the equilibrium conditions of a rigid body are applied to calculate the distance of the body CG from the pivot point. The method also allows the estimation of the CG location relative to a reference point of the subject, provided its location on the board is known. Later developments of the reaction-board method included the current force platforms that are used in biomechanics for measurement of the reaction force and moment of force vectors (and their orthogonal components) as well as of the center of pressure (COP) coordinates in static and dynamic conditions [8]. The main working principles of these platforms are based on the piezoelectric and strain gauge technology (e.g. www.kistler.com and www.amti.biz).

The purpose of the present study is to explore the potential to fiber optic sensors, namely fiber Bragg gratings (FBGs), for instrumentation of a reaction-board prototype that could be used for estimation of vertical reaction forces, moments of force and, particularly, the 2-dimension (2D) coordinates of the $\mathrm{CG}$ projection (i.e., the coordinates of the center of pressure). Additionally, the mass imbalance caused by replacement of a missing body part with an implant is addressed. In the case of the hip, for example, the metal-on-plastic implant that is usually used for hip replacement is much heavier than the bone counterpart that has been sectioned and removed. In fact, the femoral stem of the implant is typically made of a cobalt chrome alloy, having a density between $8.3 \mathrm{~g} / \mathrm{cm}^{3}$ and $9.2 \mathrm{~g} / \mathrm{cm}^{3}$, while the cortical bone density usually falls between $1.8 \mathrm{~g} / \mathrm{cm}^{3}$ and $2.1 \mathrm{~g} / \mathrm{cm}^{3}[9,10]$. Thus, the location of the CG of the bone/implant complex is expected to differ significantly from the intact femur.

\section{Experimental setup}

The experimental setup is consisted of a rigid equilateral triangular board mounted on three supports at its vertices, two of them with a cantilever instrumented with a FBG, and the other supporting the reaction platform directly (Fig. 1).

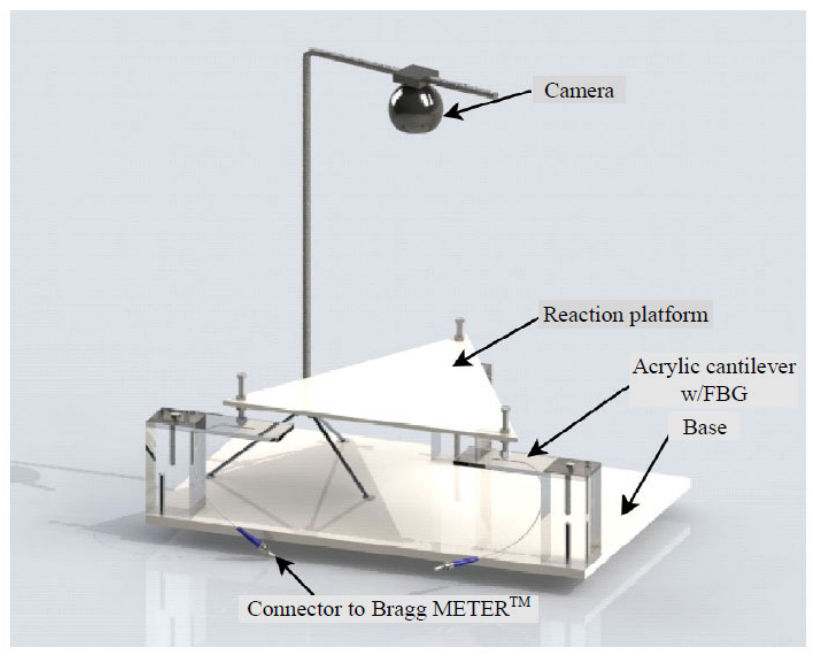

Fig. 1 Schematic representation of the setup featuring the triangular reaction-board and the cantilevers instrumented with FBGs. A camera mounted on the top of the board is used to capture an image of the object and plot the $C G$ on it automatically.

The cantilever-based design is suitable for low frequency measurements with high sensitivity and low cross-axis sensitivity. This type of sensing structure has been mainly used as an accelerometer in seismic and civil structural measurements $[11,12]$. In the present study, the FBG is glued to the bottom surface of the cantilever in order to sense strain when the reaction board is loaded with an object. The bending strain of the cantilever is transferred to the FBG which results in a wavelength shift of the Bragg's wavelength $\left(\lambda_{B}\right)$ proportional to the amount 
of strain. A prismatic cantilever beam with a rectangular section (depth $h$ and width $w$ ) is considered for calculations. The maximum vertical displacement at a distance $L$ is a load with an applied weight $P$, and is given by

$$
\delta=\frac{1}{3} \frac{P \times L}{E \times I}
$$

where $E$ is the Young's Modulus, and $I$ is the moment of inertia, given as follows:

$$
I=\frac{w \times h^{3}}{12} .
$$

For different ranges of mass over the reaction platform, the material of which the cantilevers are made can be changed, thus changing its Young's Modulus or, in alternative, the thickness of the cantilever can be changed for an already different sensitivity.

The FBGs are interrogated using a Fiber Sensing Bragg Meter (www.fibersensing.com). Additionally, a video camera is used for capturing an image of the object over the platform and drawing the CG projection point automatically. All the signals reading along with the image from the camera have been processed on LabView software to provide real time measurements.

The 2D coordinates of the $\mathrm{CG}$ are determined using the equations of equilibrium for the sum of forces and moments of forces applied to a rigid body, according to the specifications in (3) and (4) (also shown in Fig. 2).

$$
\begin{gathered}
c_{1}=\frac{\frac{c}{2}\left(-f_{R}^{A}+P-f_{R}^{B}\right)+c \times f_{R}^{B}}{P} \\
b_{1}=\frac{\frac{b}{2} \times f_{R}^{B}+b \times f_{R}^{A}}{P} .
\end{gathered}
$$

For calibration purposes, the shift in $\lambda_{B}$ is plotted against mass variation using circular calibrated masses carefully put over the reaction platform centroid. Therefore, the mass would be evenly distributed over the three vertices of the triangle according to (5). (For simplicity of this text, the term mass if often used, but the system measures forces.
The implicit relation between mass and weight should be considered along the text.)

$$
m_{x}=\frac{1}{3} \times m, x=A, B
$$

where $m$ is the mass of a giving object or body. In the previous conditions the calibration equations for estimation of the reaction forces at the vertices of the board can be obtained from

$$
m_{x}(\mathrm{~g})=\frac{\lambda_{\mathrm{FBG}}^{x}-\lambda_{B}^{x}}{\alpha_{x}}, x=A, B
$$

where $\lambda_{\mathrm{FBG}}^{x}(\mathrm{~nm})$ is the shift in the Bragg wavelength, $m_{x}(\mathrm{~g})$ is the mass at each vertex of the triangle, and $\alpha_{x}\left(\mathrm{~nm} \cdot \mathrm{g}^{-1}\right)$ is the constant of proportionality obtained from the slope of the wavelength versus mass variation plot (Fig. 3).

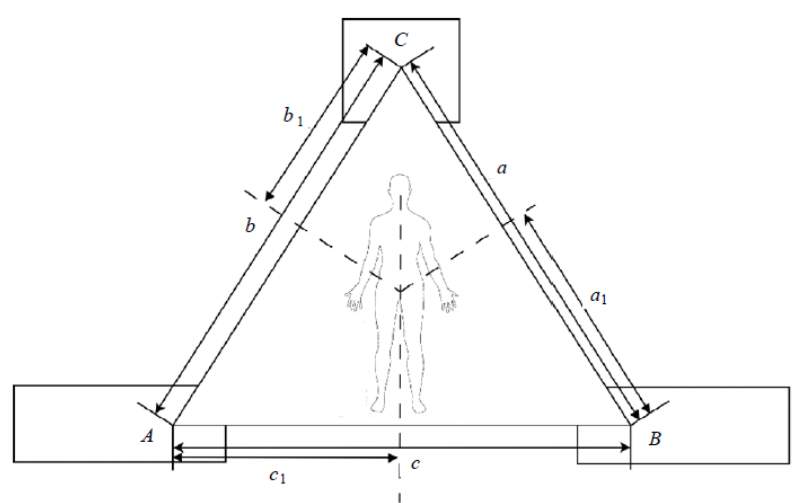

Fig. 2 Schematic representation of equilateral triangular board.

In Fig. 2, $f_{r}^{x}, x=A, B$ is the force applied on each vertex of the board and is equal to $f_{r}^{x}=m_{x} \times g, x=A, B$, where $m_{x}, x=A, B$ is the mass applied to the vertices $A$ and $B$, and $\mathrm{g}=9.81 \mathrm{~m} \cdot \mathrm{s}^{-2}$ is the acceleration of gravity; $a=b=c$ is the length of the side of the equilateral board; $p$ is the weight of the object; $c_{1}$ and $b_{1}$ are a first set of CG coordinates, natural to the reaction platform itself, that have been converted to match a point on the plot area of the image.

In this work, the setup is used to compare the location of the CG projection of a regular human synthetic femur against a femur with a hip implant. In their intact condition, both femur models have the same geometry and density distribution to enable comparisons. 


\section{Results and discussions}

In the experience, two fiber Bragg gratings at $1550 \mathrm{~nm}$ and $1555 \mathrm{~nm}$ with the same reflectivity $(\sim 50 \%)$ are used. A commercial interrogation system is used to monitor both FBGs in real time with a time acquisition of one second.

The calibration results of the reaction-board are shown in Fig. 3.

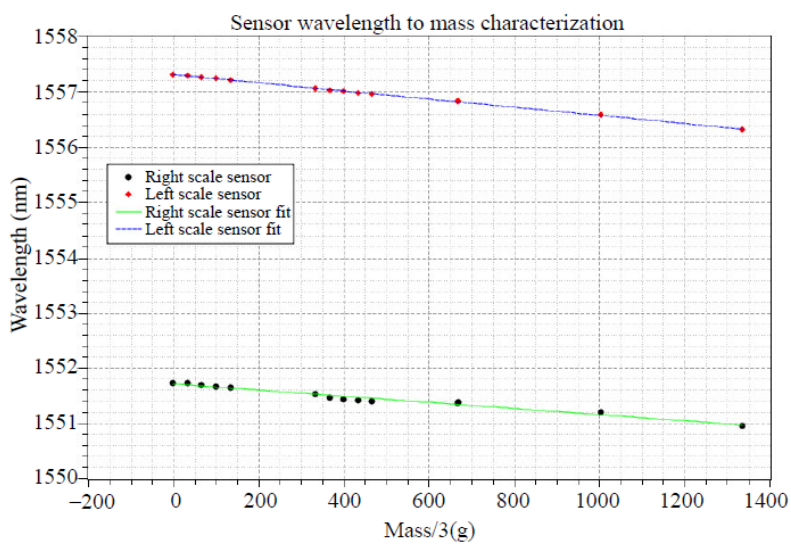

Fig. 3 Characterization of the FBGs over the applied mass. Mass (g) on the $X$ axis is $1 / 3$ of the mass of the calibrated mass used in the experiment.

As can be depicted from the previous figure, the $\lambda_{B}$ shift varies linearly with the applied mass over each cantilever. The negative slopes are due to the compression of the FBGs. The sensitivities are of $-7.36 \times 10^{-4} \mathrm{~nm} / \mathrm{g}$ and $-5.59 \times 10^{-4} \mathrm{~nm} / \mathrm{g}$ on the left and right FBGs, respectively, resulting in calibrations as follows:

$$
\begin{aligned}
& \lambda_{\mathrm{FBG}}^{A}(\mathrm{~nm})=-7.36 \times 10^{-4} \times m_{A}(\mathrm{~g})+1557.30 \\
& \lambda_{\mathrm{FBG}}^{B}(\mathrm{~nm})=-5.59 \times 10^{-4} \times m_{B}(\mathrm{~g})+1551.71 .
\end{aligned}
$$

The sensitivity is calculated separately with different masses. The sensitivity is different due to the position of the FBG glued in the cantilever. The sensitivities are obtained by a medium value of loading and unloading during the calibration.

In Fig. 4, it is depicted the measured CG projection when two cylindrical masses of $500 \mathrm{~g}$ and $1000 \mathrm{~g}$ (diameter of cylinders: $95 \mathrm{~mm}$ ) are put over the reaction platform. Measurements take place one second apart from each other, totaling 20 seconds of measurement time. It can be observed that the measurement's fluctuations have amplitude of $5 \mathrm{~mm}$ over the $Y$ axis and amplitude of $0.6 \mathrm{~mm}$ over the $X$ axis. This means $5.3 \%$ of uncertainty over the $Y$ axis and $0.6 \%$ on the $X$ axis. The explanation for the higher uncertainty over the $Y$ axis lies on the way the system is built. As the prototype is built with acrylic cantilevers, when weight is applied over them, they take a relaxation time. This time where the cantilevers are not yet fully bent by the applied weight causes the measured CG projection to slowly progress in the direction of the cantilevers.

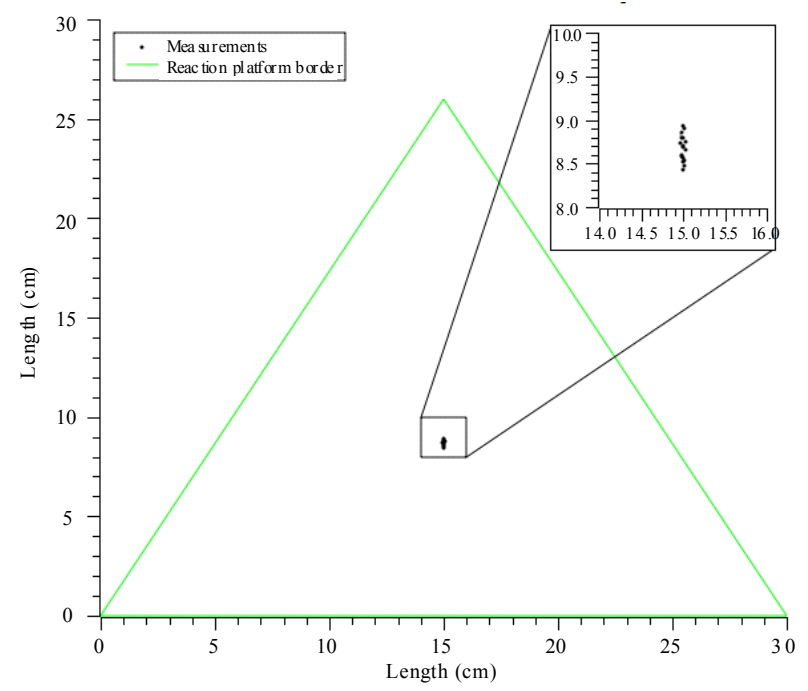

Fig. 4 Center of mass projection fluctuations over the reaction platform. Sensor behavior along time featuring two different applied weights. Measurements are made with one second intervals.

The reaction-board is used to compare the location of the CG projection of a regular human synthetic femur against a femur with a hip prosthesis. The first noticeable difference is the mass of the specimens themselves: while the femur intact model weights $569 \mathrm{~g}$, its prosthetic equivalent weights $741 \mathrm{~g}$ (plus 23\%). In fact, the metal-on-plastic implant that is usually used for hip replacement is much heavier than the bone counterpart that has been sectioned and removed. In the present case the femoral stem of the implant is made of a cobalt chrome alloy, having a density between $8.3 \mathrm{~g} / \mathrm{cm}^{3}$ and $9.2 \mathrm{~g} / \mathrm{cm}^{3}$, while the cortical bone density usually falls between $1.8 \mathrm{~g} / \mathrm{cm}^{3}$ and $2.1 \mathrm{~g} / \mathrm{cm}^{3}[9,10]$. Thus, considering the mass of the implant is mainly located at the proximal end of the femur, it is 
expected the location of the $\mathrm{CG}$ of the femur/implant complex to differ significantly from the intact femur. In fact, this feature is confirmed with measurements from the reaction-board (Fig. 5).

As expected, the $\mathrm{CG}$ of the femur/implant complex is closer to the femur head (the proximal joint or hip joint) than it is for the intact femur. The difference between the $\mathrm{CG}$ projections is around $47 \mathrm{~mm}$.

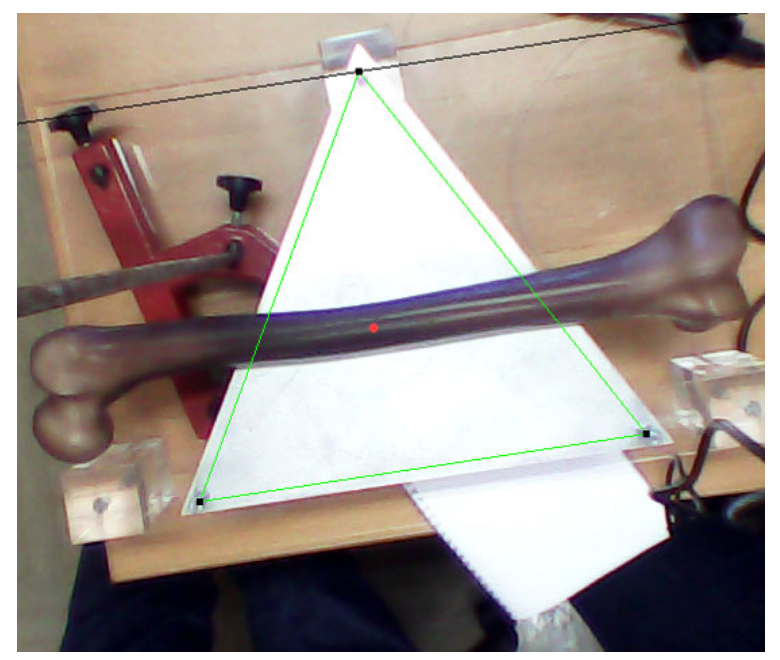

(a)

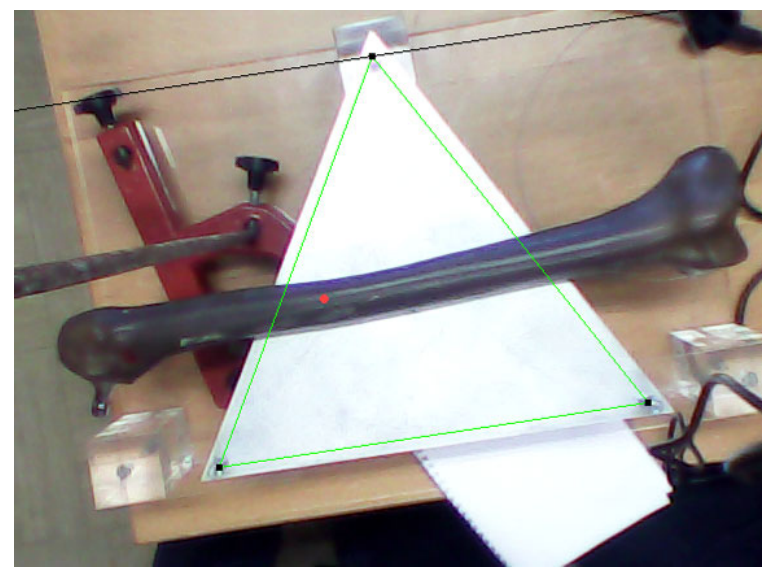

(b)

Fig. 5 Measurements of (a) femur model without prosthesis and (b) femur model with prosthesis. The dot on the bone indicates the location of the center of gravity projection.

The reference values for the thigh segment relative mass (compared to the total body mass) is about $10 \%$ and for the CG location along the longitudinal axis from the proximal joint is about $43 \%$ (of total segment length) $[3,4]$. The thigh is the second heaviest part of the body (the trunk represents around $50 \%$ of total body mass) $[3,4]$. In the present study, the CG projection of the intact femur is located around $47 \%$ of the femur length, measured from the proximal joint, approaching the reference value or the thigh. However, in the case of the femur/implant complex, the projection of the $\mathrm{CG}$ from the proximal joint is $37 \%$. Consequently, it could be argued that the Co-Cr implant modifies the limb inertial properties. Moreover, knowing the $\mathrm{CG}$ of the whole body depends on the location of the $\mathrm{CG}$ of the body parts, a significant variation of the whole-body CG location is expected in subjects with prosthetic limbs or joints.

Taking the upright posture as a reference the prosthesis will rise and shift the whole-body $\mathrm{CG}$ to the heaviest side (where the prosthesis is). Thus, it could also be hypothesized that this single disturbance could change the biomechanical behavior of the body, namely, the distribution of body weight through the feet (usually 50\% for each foot), the pattern of the ground reaction force (GRF) and the center of pressure (COP), and the trajectory of the $\mathrm{CG}$ during locomotion. Moreover, in an effort of the subject to compensate this imbalance, the body posture could also be changed (e.g., a trunk swing to the opposite side of the implant).

Thus, finding alternative designs or materials with proper mechanical properties for load bearing and densities similar to bone could be an important achievement. In fact, some alternative solutions based on less dense materials, such as magnesium and its alloys, have already been explored [9].

\section{Conclusions}

Although the resolution on the weighting machine is beyond the desirable one, noticeable change on the $\mathrm{CG}$ projection of the prosthesis is clear. Future work might imply human sized reaction platform setups for full body measurements so that the effect of prosthesis on the CG of human beings can be studded, and its implications 
discussed. It could be hypothesized that this imbalance of mass and CG distribution could change the biomechanical behaviors of the segment and the whole body, such as the distribution of body weight through the feet, the pattern of the ground reaction force and the center of pressure, and the trajectory of the CG during locomotion. On the setup itself, improvements to cantilevers quality arises, so that sensor fluctuations can be reduced, and therefore resolution can be increased as plastic relaxation phenomena are the probable issues to blame on the fluctuations of the sensor, and they have a clear negative slope on each measurement. The same method can be used for any type of body. Future work will include the development of a force platform based on fiber optic sensors intended for calculation of the tree components of the reaction force and the tree moments of force along with the center of pressure mapping in the surface of the platform.

Open Access This article is distributed under the terms of the Creative Commons Attribution 4.0 International License (http://creativecommons.org/ licenses/by/4.0/), which permits unrestricted use, distribution, and reproduction in any medium, provided you give appropriate credit to the original author(s) and the source, provide a link to the Creative Commons license, and indicate if changes were made.

\section{References}

[1] R. N. Hinrichs, "Adjustments to the segment center of mass proportions of Clauser et al. (1969)," Journal of Biomechanics, 1990, 23(9): 949-951.

[2] E. M. Gutierrez-Farewik, A. Bartonek, and H. Saraste, "Comparison and evaluation of two common methods to measure center of mass displacement in three dimensions during gait," Human Movement Science, 2006, 25(2): 238-256.

[3] B. M. Nigg and W. Herzog, Biomechanics of the musculo-skeletal system. West Sussex, England: John Wiley \& Sons, 1999: 1-686.

[4] D. A. Winter, Biomechanics and motor control of human movement. New Jersey, USA: John Wiley \& Sons, 2009: 1-358.

[5] E. Reynolds and R. W. Lovett, "A method of determining the position of the centre of gravity in its relation to certain bony landmarks in the erect position," American Journal of Physiology, 1909, 24(2): 286-293.

[6] E. Reynolds and R. W. Lovett, “An experimental study of certain phases of chronic backache: a combined gynecologic and orthopedic investigation," Journal of the American Medical Association, 1910, Liv(13): 1033-1043.

[7] W. Mckino, C. Hartford, Z. L. Di, J. van Schalkwyk, D. Veliotes, A. Hofmeyr, et al., "The agreement between reaction-board measurements and kinematic estimation of adult male human whole body centre of mass location during running," Physiological Measurement, 2004, 25(6): 1339-1354.

[8] R. P. Silveira, P. Stergiou, F. P. Carpes, F. A. D. S. Castro, L. Katz, and D. J. Stefanyshyn, "Validity of a portable force platform for assessing biomechanical parameters in three different tasks," Sports Biomechanics, 2017, 16(2): 177-186.

[9] M. P. Staiger, A. M. Pietak, J. Huadmai, and G. Dias, "Magnesium and its alloys as orthopedic biomaterials: a review," Biomaterials, 2006, 27(9): 1728-1734.

[10] D. A. Winter, Biomechanicsand motor control of human movement. New Jersey, USA: John Wiley \& Sons, 2009: 1-358.

[11] M. D. Todd, G. A. Johnson, B. A. Althouse, and S. T. Vohra, "Flexural beam-based fiber Bragg grating accelerometers," Photonics Technology Letters, 1998, 10(11): 1605-1607.

[12] T. A. Berkoff and A. D. Kersey, "Experimental demonstration of a fiber Bragg grating accelerometer," Photonics Technology Letters, 8(12): 1677-1679. 